\title{
The return of a static universe and the end of cosmology
}

\author{
Lawrence M. Krauss • Robert J. Scherrer
}

Published online: 28 June 2007

(C) Springer Science+Business Media, LLC 2007

\begin{abstract}
We demonstrate that as we extrapolate the current $\Lambda \mathrm{CDM}$ universe forward in time, all evidence of the Hubble expansion will disappear, so that observers in our "island universe" will be fundamentally incapable of determining the true nature of the universe, including the existence of the highly dominant vacuum energy, the existence of the $\mathrm{CMB}$, and the primordial origin of light elements. With these pillars of the modern Big Bang gone, this epoch will mark the end of cosmology and the return of a static universe. In this sense, the coordinate system appropriate for future observers will perhaps fittingly resemble the static coordinate system in which the de Sitter universe was first presented.
\end{abstract}

\section{Introduction}

Shortly after Einstein's development of general relativity, the Dutch astronomer Willem de Sitter proposed a static model of the universe containing no matter, which he thought might be a reasonable approximation to our low density universe. One can define a coordinate system in which the de Sitter metric takes a static form by defining de Sitter spacetime with a cosmological constant $\Lambda$ as a four dimensional hyperboloid $\mathcal{S}_{\Lambda}: \eta_{A B} \xi^{A} \xi^{B}=-R^{2}, R^{2}=3 \Lambda^{-1}$ embedded in a $5 d$ Minkowski spacetime with

Fifth Award in the 2007 Essay Competition of the Gravity Research Foundation.

L. M. Krauss $(\varangle)$

Department of Physics, Case Western Reserve University, Cleveland, OH 44106, USA

e-mail: krauss@cwru.edu

L. M. Krauss · R. J. Scherrer

Department of Physics and Astronomy, Vanderbilt University, Nashville, TN 37235, USA

e-mail: robert.scherrer@vanderbilt.edu 
$d s^{2}=\eta_{A B} d \xi^{A} d \xi^{B}$, and $\left(\eta_{A B}\right)=\operatorname{diag}(1,-1,-1,-1,-1), A, B=0, \ldots, 4$. The static form of the de Sitter metric is then

$$
d s_{s}^{2}=\left(1-r_{s}^{2} / R^{2}\right) d t_{s}^{2}-\frac{d r_{s}^{2}}{1-r_{s}^{2} / R^{2}}-r_{s}^{2} d \Omega^{2},
$$

which can be obtained by setting $\xi^{0}=\left(R^{2}-r_{s}^{2}\right)^{1 / 2} \sinh \left(t_{s} / R\right), \xi^{1}=r_{s} \sin \theta \cos \varphi$, $\xi^{2}=r_{s} \sin \theta \sin \varphi, \xi^{3}=r_{s} \cos \theta, \xi^{4}=\left(R^{2}-r_{s}^{2}\right)^{1 / 2} \cosh \left(t_{s} / R\right)$. In this case the metric only corresponds to the section of de Sitter space within a cosmological horizon at $R=r-s$.

In fact de Sitter's model wasn't globally static, but eternally expanding, as can be seen by a coordinate transformation which explicitly incorporates the time dependence of the scale factor $R(t)=\exp (H t)$. While spatially flat, it actually incorporated Einstein's cosmological term, which is of course now understood to be equivalent to a vacuum energy density, leading to a redshift proportional to distance.

The de Sitter model languished for much of the last century, once the Hubble expansion had been discovered, and the cosmological term abandoned. However, all present observational evidence is consistent with a $\Lambda \mathrm{CDM}$ flat universe consisting of roughly $30 \%$ matter (both dark matter and baryonic matter) and 70\% dark energy [1-3], with the latter having a density that appears constant with time. All cosmological models with a non-zero cosmological constant will approach a de Sitter universe in the far future, and many of the implications of this fact have been explored in the literature [4-13].

Here we re-examine the practical significance of the ultimate de Sitter expansion and point out a new eschatological physical consequence: from the perspective of any observer within a bound gravitational system in the far future, the static version of de Sitter space outside of that system will eventually become the appropriate physical coordinate system. Put more succinctly, in a time comparable to the age of the longest lived stars, observers will not be able to perform any observation or experiment that infers either the existence of an expanding universe dominated by a cosmological constant, or that there was a hot Big Bang. Observers will be able to infer a finite age for their island universe, but beyond that cosmology will effectively be over. The static universe, with which cosmology at the turn of the last century began, will have returned with a vengeance.

Modern cosmology is built on integrating general relativity and three observational pillars: the observed Hubble expansion, detection of the cosmic microwave background radiation, and the determination of the abundance of elements produced in the early universe. We describe next in detail how these observables will disappear for an observer in the far future, and how this will be likely to affect the theoretical conclusions one might derive about the universe.

\section{The disappearance of the Hubble expansion}

The most basic component of modern cosmology is the expansion of the universe, firmly established by Hubble in 1929. Currently, galaxies and galaxy clusters are 
gravitationally bound and have dropped out of the Hubble flow, but structures on larger length scales are observed to obey the Hubble expansion law. Now consider what happens in the far future of the universe. Both analytic [7] and numerical [10] calculations indicate that the Local Group remains gravitationally bound in the face of the accelerated Hubble expansion. All more distant structures will be driven outside of the de Sitter event horizon in a timescale on the order of 100 billion years ([4], see also Refs. [8,9]). While objects will not be observed to cross the event horizon, light from them will be exponentially redshifted, so that within a time frame comparable to the longest lived main sequence stars all objects outside of our local cluster will truly become invisible [4].

Since the only remaining visible objects will in fact be gravitationally bound and decoupled from the underlying Hubble expansion, any local observer in the far future will see a single galaxy (the merger product of the Milky Way and Andromeda and other remnants of the Local Group) and will have no observational evidence of the Hubble expansion. Lacking such evidence, one may wonder whether such an observer will postulate the correct cosmological model. We would argue that in fact, such an observer will conclude the existence of a static "island universe," precisely the standard model of the universe ca. 1900.

This will be true in spite of the fact that the dominant energy in this universe will not be due to matter, but due to dark energy, with $\rho_{M} / \rho_{\Lambda} \sim 10^{-12}$ inside the horizon volume [9]. The irony, of course, is that the denizens of this static universe will have no idea of the existence of the dark energy, much less of its magnitude, since they will have no probes of the length scales over which $\Lambda$ dominates gravitational dynamics. It appears that dark energy is undetectable not only in the limit where $\rho_{\Lambda} \ll \rho_{M}$, but also when $\rho_{\Lambda} \gg \rho_{M}$.

Even if there were no direct evidence of the Hubble expansion, we might expect three other bits of evidence, two observational and one theoretical, to lead physicists in the future to ascertain the underlying nature of cosmology. However, we next describe how this is unlikely to be the case.

\section{Vanishing CMB}

The existence of a Cosmic Microwave Background was the key observation that convinced most physicists and astronomers that there was in fact a hot big bang, which essentially implies a Hubble expansion today. But even if skeptical observers in the future were inclined to undertake a search for this afterglow of the Big Bang, they would come up empty-handed. At $t \approx 100 \mathrm{Gyr}$, the peak wavelength of the cosmic microwave background will be redshifted to roughly $\lambda \approx 1 \mathrm{~m}$, or a frequency of roughly $300 \mathrm{MHz}$. While a uniform radio background at this frequency would in principle be observable, the intensity of the CMB will also be redshifted by about 12 orders of magnitude. At much later times, the CMB becomes unobservable even in principle, as the peak wavelength is driven to a length larger than the horizon [4]. Well before then, however, the microwave background peak will redshift below the plasma frequency of the interstellar medium, and so will be screened from any observer within 
the galaxy. Recall that the plasma frequency is given by

$$
v_{p}=\left(\frac{n_{e} e^{2}}{\pi m_{e}}\right)^{1 / 2}
$$

where $n_{e}$ and $m_{e}$ are the electron number density and mass, respectively. Observations of dispersion in pulsar signals give [14] $n_{e} \approx 0.03 \mathrm{~cm}^{-3}$ in the interstellar medium, which corresponds to a plasma frequency of $v_{p} \approx 1 \mathrm{kHz}$, or a wavelength of $\lambda_{p} \approx 3 \times 10^{7} \mathrm{~cm}$. This corresponds to an expansion factor $\sim 10^{8}$ relative to the present-day peak of the CMB. Assuming an exponential expansion, dominated by dark energy, this expansion factor will be reached when the universe is less than 50 times its present age, well below the lifetime of the longest-lived main sequence stars.

After this time, even if future residents of our island universe set out to measure a universal radiation background, they would be unable to do so. The wealth of information about early universe cosmology that can be derived from fluctuations in the $\mathrm{CMB}$ would be even further out of reach.

\section{General relativity gives no assistance}

We may assume that theoretical physicists in the future will infer that gravitation is described by general relativity, using observations of planetary dynamics, and groundbased tests of such phenomena as gravitational time dilation. Will they then not be led to a Big Bang expansion, and a beginning in a Big Bang singularity, independent of data, as Lemaitre was? Indeed, is not a static universe incompatible with general relativity?

The answer is no. The inference that the universe must be expanding or contracting is dependent upon the cosmological hypothesis that we live in an isotropic and homogeneous universe. For future observers, this will manifestly not be the case. Outside of our local cluster, the universe will appear to be empty and static. Nothing is inconsistent with the temporary existence of a non-singular isolated self-gravitating object in such a universe, governed by general relativity. Physicists will infer that this system must ultimately collapse into a future singularity, but only as we presently conclude our galaxy must ultimately coalesce into a large black hole. Outside of this region, an empty static universe can prevail.

While physicists in the island universe will therefore conclude that their island has a finite future, the question will naturally arise as to whether it had a finite beginning. As we next describe, observers will in fact be able to determine the age of their local cluster, but not the nature of the beginning.

\section{Polluted elemental abundances}

The theory of Big Bang Nucleosynthesis reached a fully-developed state [15] only after the discovery of the CMB (despite early abortive attempts by Gamow and his collaborators [16]). Thus, it is unlikely that the residents of the static universe would have any 
motivation to explore the possibility of primordial nucleosynthesis. However, even if they did, the evidence for BBN rests crucially on the fact that relic abundances of deuterium remain observable at the present day, while helium-4 has been enhanced by only a few percent since it was produced in the early universe. Extrapolating forward by $100 \mathrm{Gyr}$, we expect significantly more contamination of the helium- 4 abundance, and concomitant destruction of the relic deuterium. It has been argued [18] that the ultimate extrapolation of light elemental abundances, following many generations of stellar evolution, is a mass fraction of helium given by $Y=0.6$. The primordial helium mass fraction of $Y=0.25$ will be a relatively small fraction of this abundance. It is unlikely that much deuterium could survive this degree of processing. Of course, the current "smoking gun" deuterium abundance is provided by Lyman- $\alpha$ absorption systems, back-lit by QSOs (see, e.g., Ref. [19]). Such systems will be unavailable to our observers of the future, as both the QSOs and the Lyman- $\alpha$ systems will have redshifted outside of the horizon.

Astute observers will be able to determine a lower limit on the age of their system, however, using standard stellar evolution analyses of their own local stars. They will be able to examine the locus of all stars and extrapolate to the oldest such stars to estimate a lower bound on the age of the galaxy. They will be able to determine an upper limit as well, by determining how long it would take for all of the observed helium to be generated by stellar nucleosynthesis. However, without any way to detect primordial elemental abundances, such as the aforementioned possibility of measuring deuterium in distant intergalactic clouds that currently absorb radiation from distant quasars and allow a determination of the deuterium abundance in these pre-stellar systems, and with the primordial helium abundance dwarfed by that produced in stars, inferring the original BBN abundances will be difficult, and probably not well motivated.

Thus, while physicists of the future will be able to infer that their island universe has not been eternal, it is unlikely that they will be able to infer that the beginning involved a Big Bang.

\section{Conclusion}

The remarkable cosmic coincidence that we happen to live at the only time in the history of the universe when the magnitude of dark energy and dark matter densities are comparable has been a source of great current speculation, leading to a resurgence of interest in possible anthropic arguments limiting the value of the vacuum energy (see, e.g., Refs. [20,21]). But this coincidence endows our current epoch with another special feature, namely that we can actually infer both the existence of the cosmological expansion, and the existence of dark energy. Thus, we live in a very special time in the evolution of the universe: the time at which we can observationally verify that we live in a very special time in the evolution of the universe!

Observers when the universe was an order of magnitude younger would not have been able to discern any effects of dark energy on the expansion, and observers when the universe is more than an order of magnitude older will be hard pressed to know that they live in an expanding universe at all, or that the expansion is dominated by dark energy. By the time the longest lived main sequence stars are nearing the end of their 
lives, for all intents and purposes, the universe will appear static, and all evidence that now forms the basis of our current understanding of cosmology will have disappeared.

Note added in proof: After this paper was submitted we learned of a prescient 1987 paper [22], written before the discovery of dark energy and other cosmological observables that are central to our analysis, which nevertheless raised the general question of whether there would be epochs in the Universe when observational cosmology, as we now understand it, would not be possible.

Acknowledgments L.M.K. and R.J.S. were supported in part by the Department of Energy.

\section{References}

1. Krauss, L.M., Turner, M.S.: Gen. Rel. Grav. 27, 1137 (1995)

2. Perlmutter, S., et al.: Astrophysics 517, 565 (1999)

3. Reiss, A.G., et al.: Astron. J. 116, 1009 (1998)

4. Krauss, L.M., Starkman, G.D.: Astrophysics 531, 22 (2000)

5. Starobinsky, A.A.: Grav. Cosmol. 6, 157 (2000)

6. Gudmundsson, E.H., Bjornsson, G.: Astrophysics 565, 1 (2002)

7. Loeb, A.: Phys. Rev. D 65, 047301 (2002)

8. Chiueh, T., He, X.-G.: Phys. Rev. D 65, 123518 (2002)

9. Busha, M.T., Adams, F.C., Wechsler, R.H., Evrard, A.E.: Astrophysics 596, 713 (2003)

10. Nagamine, K., Loeb, A.: New Astron. 8, 439 (2003)

11. Nagamine, K., Loeb, A.: New Astron. 9, 573 (2004)

12. Heyl, J.S.: Phys. Rev. D 72, 107302 (2005)

13. Krauss, L.M., Scherrer, R.J.: Phys. Rev. D 75, 083524 (2007)

14. Tielens, A.G.G.M.: The Physics and Chemistry of the Interstellar Medium. Cambridge University Press, Cambridge (2005)

15. Wagoner, R.V., Fowler, W.A., Hoyle, F.: Astrophysics 148, 3 (1967)

16. Alpher, R.A., Bethe, H., Gamow, G.: Phys. Rev. 73, 803 (1948)

17. Alpher, R.A., Follin, J.W., Herman, R.C.: Phys. Rev. 92, 1347 (1953)

18. Adams, F.C., Laughlin, G.: Rev. Mod. Phys. 69, 337 (1997)

19. Kirkman, D., Tytler, D., Suzuki, N., O’Meara, J.M., Lubin, D.: Ap. J. Suppl. 149, 1 (2003)

20. Weinberg, S.: Phys. Rev. Lett. 59, 2607 (1987)

21. Garriga, J., Livio, M., Vilenkin, A.: Phys. Rev. D 61, 023503 (2000)

22. Rothman, T., Ellis, G.F.R.: Observatory 107, 24 (1987) 